New Theorry

ISSN: 2149-1402
37 (2021) 26-34

Journal of New Theory

https://dergipark.org.tr/en/pub/jnt

Open Access

\title{
The Transmittal-Characteristic Function of Three-Interval Periodic Sturm-Liouville Problem with Transmission Conditions
}

\author{
Kadriye Aydemir ${ }^{1}$ (D) Oktay Mukhtarov $^{2}$
}

\author{
Article History \\ Received: 03 Nov 2021 \\ Accepted: 28 Dec 2021 \\ Published: 31 Dec 2021 \\ 10.53570/jnt.1018600 \\ Research Article
}

\begin{abstract}
In this paper, we study the periodic Sturm-Liouville problem, defined on three non-intersecting intervals with four supplementary conditions which are imposed at two internal points of interaction, the so-called transmission conditions. We first prove that the eigenvalues are real and the system of eigenfunctions is an orthogonal system. Secondly, some auxiliary initial-value problems are defined and transmittal-characteristic function is constructed in terms of solutions of these initial-value problems. Finally, we establish that the eigenvalues of the considered problem are the zeros of the transmittal-characteristic function.
\end{abstract}

Keywords - Periodic transmittal Sturm-Liouville problem, characteristic function, spectrum

Mathematics Subject Classification (2020) - 34B24, 34L15

\section{Introduction}

This paper is aimed at studying a discontinuous spectral problem consisting of the three-interval Sturm-Liouville equation

$$
\left.\left.\Xi_{\lambda} y:=-y^{\prime \prime}(x)+q(x) y=\lambda y(x) \quad x \in[\urcorner_{1}, c_{1}\right) \cup\left(c_{1}, c_{2}\right) \cup\left(c_{2},\right\urcorner_{2}\right]
$$

the periodic boundary conditions

$$
\begin{aligned}
\ell_{1} y & \left.\left.:=y(\urcorner_{1}\right)-y(\urcorner_{2}\right)=0 \\
\ell_{2} y & \left.\left.:=y^{\prime}(\urcorner_{1}\right)-y^{\prime}(\urcorner_{2}\right)=0
\end{aligned}
$$

and supplementary transmission conditions, which are imposed at the points of interaction $c_{1}$ and $c_{2}$, given by

$$
\begin{aligned}
& \ell_{3} y:=y\left(c_{1}^{-}\right)-y\left(c_{1}^{+}\right)=0 \\
& \ell_{4} y:=y^{\prime}\left(c_{1}^{-}\right)-y^{\prime}\left(c_{1}^{+}\right)=0
\end{aligned}
$$

and

$$
\begin{aligned}
\ell_{5} y & :=y\left(c_{2}^{-}\right)-\beta y\left(c_{2}^{+}\right)=0 \\
\ell_{6} y & :=y^{\prime}\left(c_{2}^{-}\right)-\frac{1}{\beta} y^{\prime}\left(c_{2}^{+}\right)=0
\end{aligned}
$$

respectively, where $q(x)$ is a real-valued function, $\lambda \in C$ is a complex spectral parameter and the coefficient $\beta \neq 0$ is real numbers. In investigating the periodic flow in a rod, C. Sturm and J. Liouville

\footnotetext{
${ }^{1}$ kadriyeaydemr@gmail.com (Corresponding Author); ${ }^{2}$ omukhtarov@yahoo.com

${ }^{1}$ Department of Mathematics, Faculty of Arts and Sciences, Amasya University, Amasya, Turkey

${ }^{2}$ Department of Mathematics, Faculty of Arts and Sciences, Tokat Gaziosmanpaşa University, Tokat, Turkey
} 
in the first half of the 19th century were led to the definition of class of Sturm-Liouville problems consisting of self-adjoint linear differential equation of the form

$$
-\frac{d}{d x}\left(K(x) \frac{d F(x)}{d x}\right)+\ell(x) F(x)=\lambda q(x) F(x) \quad \text { for } x \in[a, b]
$$

together with the boundary conditions of the form

$$
\begin{aligned}
& K(a) F^{\prime}(a)-h F(a)=0 \\
& K(b) F^{\prime}(b)-H F(b)=0
\end{aligned}
$$

They obtained some results that characterized by the general and qualitative nature of the solutions. They also proved that there exists infinitely countable number of values $\lambda_{1}, \lambda_{2}, \ldots$ of spectral parameter $\lambda$ with the corresponding non-trivial solutions $F_{1}(x), F_{2}(x), \ldots$, the so- called eigenfunctions, and discussed the qualitative behavior of these eigenvalues and eigenfunctions, such as the asymptotics of eigenvalues, the zeros of the eigenfunctions that could be used in a variety of physical situations. These results have inspired much of branches of modern analysis and spectral theory of linear differential and integral operators, and continue to do so. The existence of periodic and oscillatory eigenfunctions important in the spectral theory of differential operators. We know that periodic boundary value problems of Sturm-Liouville type have been widely investigated due to their application in physics and engineering. For example, consider the heated string bent into a circle. Since the two ends of this string are physically the same, we would expect that the temperature and the temperature gradient to be equal at these endpoints. This situation is modelled by boundary conditions of the form

$$
u(a)=u(b), \quad u^{\prime}(a)=u^{\prime}(b)
$$

which are called periodic boundary conditions.

Periodic Sturm-Liouville problems for various type differential equations have been studied extensively in the literature( see, for example, [1-5] and references therein). In the paper [2], the authors considered the problem

$$
\left\{\begin{array}{l}
-u^{\prime \prime}+h(s) u=\lambda g(s, u), \quad 0 \leq s \leq \pi \\
u(0)=u(2 \pi), \quad u^{\prime}(0)=u^{\prime}(2 \pi)
\end{array}\right.
$$

and

$$
\left\{\begin{array}{l}
u^{\prime \prime}+h(s) u=\lambda g(s, u), \quad 0 \leq s \leq \pi \\
u(0)=u(2 \pi), \quad u^{\prime}(0)=u^{\prime}(2 \pi)
\end{array}\right.
$$

where $h \in L^{1}(0,2 \pi), g:[0,2 \pi] \times \mathbb{R}^{+} \rightarrow \mathbb{R}^{+}$is continuous, $\lambda$ is a positive parameter. In the work [3], a new existence theorems for a nonlinear periodic boundary value problem of first-order differential equations with impulses are established. In the article [4], the topological degree theory is applied to show the existence of positive solutions to the periodic Sturm-Liouville problem. In the paper [5] the eigenvalues of regular periodic and semi periodic Sturm-Liouville problems are considered. Binding and Rynne [6] considered the nonlinear Sturm-Liouville problem

$$
\left\{\begin{array}{l}
(-\rho y)^{\prime \prime}+\varrho y=a y^{+}-b y^{-}+\lambda y \\
y(0)=y(2 \pi), \quad(\rho y)^{\prime}(0)=(\rho y)^{\prime}(2 \pi)
\end{array}\right.
$$

where $\frac{1}{\rho}, \varrho \in L^{1}(0,2 \pi)$ with $\rho>0$ on $(0,2 \pi), a, b \in L^{1}(0,2 \pi) \lambda$ is a real parameter, and $y^{ \pm}(t)=$ $\max \{ \pm y(t), 0\}$ for $t \in[0,2 \pi]$. It is showed that a sequence of half-eigenvalues exists and obtained degree theoretic properties associated with set of half-eigenvalues. In recent years, many spectral properties of periodic Sturm-Liouville problems have been studied and many techniques have been developed by many authors(see [7-16]).

Boundary value problems including transmission conditions appears in many fields of natural sciences. Recently, such type of transmission problems have been an important topic in theoretical and applied mathematics (see, [17-28]). In this study we will investigate some basic spectral properties of a new type periodic Sturm-Liouville problems. Namely, the differential equations are defined on 
three separated subintervals and the boundary conditions are set not only at the ends of the considered interval, but also at the internal points of interaction. We proved that the eigenvalues are real and the eigenfunctions belongs to distinct eigenvalues are orthogonal. We also defined a new type of characteristic function, the roots of which coincide with the eigenvalues.

\section{Eigenvalues and Eigenfunctions of the Considered problem}

Theorem 2.1. The eigenvalues of the three-interval periodic problem (1) - (7) are all real.

Proof. Let $\lambda$ be an eigenvalue of the considered problem $(1)-(7)$ with an eigenfunction $\psi$. Taking the complex conjugate and note that the coefficient $\beta$ is real, we arrive at

$$
\begin{gathered}
\overline{-\psi^{\prime \prime}(x)}=\overline{\lambda \psi}(x) \\
\overline{\left.\psi(\urcorner_{1}\right)}=\overline{\left.\psi(\urcorner_{2}\right)}, \quad \overline{\left.\psi^{\prime}(\urcorner_{1}\right)}=\overline{\left.\psi^{\prime}(\urcorner_{2}\right)} \\
\overline{\psi\left(c_{1}^{-}\right)}=\overline{\psi\left(c_{1}^{+}\right)}, \quad \overline{\psi^{\prime}\left(c_{1}^{-}\right)}=\overline{\psi^{\prime}\left(c_{1}^{+}\right)} \\
\overline{\psi\left(c_{2}^{-}\right)}=\beta \overline{\psi\left(c_{2}^{+}\right)}, \quad \overline{\psi^{\prime}\left(c_{2}^{-}\right)}=\frac{1}{\beta} \overline{\psi^{\prime}\left(c_{2}^{+}\right)}
\end{gathered}
$$

This implies that $(\bar{\lambda}, \bar{\psi})$ is also an eigen pair for the problem $(1)-(7)$. By the previous theorem we have

$$
\begin{aligned}
0 & =(\lambda-\bar{\lambda})\left(\int_{\urcorner_{1}}^{c_{1}^{-}} \overline{\psi(x)} \psi(x) d x+\int_{c_{1}^{+}}^{c_{2}^{-}} \overline{\psi(x)} \psi(x) d x+\int_{c_{2}^{+}}^{\urcorner_{2}} \overline{\psi(x)} \psi(x) d x\right) \\
& =(\lambda-\bar{\lambda})\left(\int_{\urcorner_{1}}^{c_{1}^{-}}|\psi(x)|^{2} d x+\int_{c_{1}^{+}}^{c_{2}^{-}}|\psi(x)|^{2} d x+\int_{c_{2}^{+}}^{\urcorner_{2}}|\psi(x)|^{2} d x\right)
\end{aligned}
$$

Since $\psi$, being an eigenfunction, is not identically equal to zero on [\rceil$\left.\left._{1}, c_{1}\right) \cup\left(c_{1}, c_{2}\right) \cup\left(c_{2},\right\urcorner_{2}\right]$

$$
\int_{\tau_{1}}^{c_{1}^{-}}|\psi(x)|^{2} d x+\int_{c_{1}^{+}}^{c_{2}^{-}}|\psi(x)|^{2} d x+\int_{c_{2}^{+}}^{7_{2}}|\psi(x)|^{2} d x>0
$$

So, $\lambda=\bar{\lambda}$. Thus $\lambda$ is real. The proof is complete.

Remark 2.2. Since all eigenvalues of the considered problem (1) - (7) are real, without loss of generality we can now assume that the corresponding eigenfunctions are also real-valued.

Theorem 2.3. Let $\lambda_{k}$ and $\lambda_{r}$ are two distinct eigenvalues of the problem $(1)-(7)$ on [\rceil$\left._{1}, c_{1}\right) \cup$ $\left.\left(c_{1}, c_{2}\right) \cup\left(c_{2},\right\urcorner_{2}\right]$, then their corresponding eigenfunctions $\psi_{k}$ and $\psi_{r}$ satisfy the following equality

$$
\int_{\tau_{1}}^{c_{1}^{-}} \psi_{k}(x) \psi_{r}(x) d x+\int_{c_{1}^{+}}^{c_{2}^{-}} \psi_{k}(x) \psi_{r}(x) d x+\int_{c_{2}^{+}}^{7_{2}} \psi_{k}(x) \psi_{r}(x) d x=0
$$

that is the eigenfunctions $\psi_{k}$ and $\psi_{r}$ are orthogonal in the Hilbert space $\left.L_{2}\left(\left((\urcorner_{1}, c_{1}\right) \oplus\left(c_{1}, c_{2}\right) \oplus\left(c_{2},\right\urcorner_{2}\right)\right)$. Proof. Since $\psi_{k}$ and $\psi_{r}$ are eigenfunctions corresponding to the eigenvalues $\lambda_{k}$ and $\lambda_{r}$ respectively, we have

$$
-\psi_{k}^{\prime \prime}(x)=\lambda_{k} \psi_{k}(x)
$$


and

$$
-\psi_{r}^{\prime \prime}(x)=\lambda_{r} \psi_{r}(x)
$$

Multiplying (17) by $\psi_{r}$ and (18) by $\psi_{k}$, then subtracting we get

$$
\begin{aligned}
& \left(\lambda_{k}-\lambda_{r}\right)\left(\int_{\urcorner_{1}}^{c_{1}^{-}} \psi_{k}(x) \psi_{r}(x) d x+\int_{c_{1}^{+}}^{c_{2}^{-}} \psi_{k}(x) \psi_{r}(x) d x+\int_{c_{2}^{+}}^{\urcorner_{2}} \psi_{k}(x) \psi_{r}(x) d x\right) \\
& =\left.\left(\psi_{k}^{\prime} \psi_{r}-\psi_{r}^{\prime} \psi_{k}\right)\right|_{\urcorner_{1}} ^{c_{1}^{-}}+\left.\left(\psi_{k}^{\prime} \psi_{r}-\psi_{r}^{\prime} \psi_{k}\right)\right|_{c_{1}^{+}} ^{c_{2}^{-}}+\left.\left(\psi_{k}^{\prime} \psi_{r}-\psi_{r}^{\prime} \psi_{k}\right)\right|_{c_{2}^{+}} ^{\urcorner_{2}} .
\end{aligned}
$$

By using the boundary and transmission conditions we find

$$
\left(\lambda_{k}-\lambda_{r}\right)\left(\int_{\urcorner_{1}}^{c_{1}^{-}} \psi_{k}(x) \psi_{r}(x) d x+\int_{c_{1}^{+}}^{c_{2}^{-}} \psi_{k}(x) \psi_{r}(x) d x+\int_{c_{2}^{+}}^{\urcorner_{2}} \psi_{k}(x) \psi_{r}(x) d x\right)=0
$$

Since $\lambda_{k} \neq \lambda_{r}$ we get the equality (16).

Theorem 2.4. The periodic problem $(1)-(7)$ is self-adjoint.

Proof. Consider the periodic Sturm-Liouville problem $(1)-(7)$. Let $u, \vartheta \in C_{2}\left((\urcorner_{1}, c_{1}\right) \oplus\left(c_{1}, c_{2}\right) \oplus$ $\left.\left.\left(c_{2},\right\urcorner_{2}\right)\right)$ that satisfies the periodic eigenvalue problem $(1)-(7)$. We shall prove that

$$
\int_{\urcorner_{1}}^{c_{1}^{-}}\left[u \Xi_{\lambda} \vartheta-\vartheta \Xi_{\lambda} u\right] d x+\int_{c_{1}^{+}}^{c_{2}^{-}}\left[u \Xi_{\lambda} \vartheta-\vartheta \Xi_{\lambda} u\right] d x+\int_{c_{2}^{+}}^{\urcorner_{2}}\left[u \Xi_{\lambda} \vartheta-\vartheta \Xi_{\lambda} u\right] d x=0 .
$$

By using the definition of the differential operator $\Xi_{\lambda}$ we can show that

$$
\vartheta \Xi_{\lambda} u-u \Xi_{\lambda} \vartheta=\frac{d}{d x}\left(\vartheta u^{\prime}-u \vartheta^{\prime}\right)
$$

Now integrating by parts over [\rceil$\left.\left._{1}, c_{1}\right) \cup\left(c_{1}, c_{2}\right) \cup\left(c_{2},\right\urcorner_{2}\right]$ we obtain

$$
\begin{aligned}
& \int_{\tau_{1}}^{c_{1}^{-}}\left[\vartheta \Xi_{\lambda} u-u \Xi_{\lambda} \vartheta\right] d x+\int_{c_{1}^{+}}^{c_{2}^{-}}\left[\vartheta \Xi_{\lambda} u-u \Xi_{\lambda} \vartheta\right] d x+\int_{c_{2}^{+}}^{7_{2}}\left[\vartheta \Xi_{\lambda} u-u \Xi_{\lambda} \vartheta\right] d x \\
& =\left.\left(\vartheta u^{\prime}-u \vartheta^{\prime}\right)\right|_{7_{1}} ^{c_{1}^{-}}+\left.\left(\vartheta u^{\prime}-u \vartheta^{\prime}\right)\right|_{c_{1}^{+}} ^{c_{2}^{-}}+\left.\left(\vartheta u^{\prime}-u \vartheta^{\prime}\right)\right|_{c_{2}^{+}} ^{7_{2}} .
\end{aligned}
$$

To satisfy the conditions $(2)-(3)$ we get

$$
\left.\left.\left.\left.u(\urcorner_{1}\right)=u(\urcorner_{2}\right), u^{\prime}(\urcorner_{1}\right)=u^{\prime}(\urcorner_{2}\right)
$$

and

$$
\left.\left.\left.\left.\vartheta(\rceil_{1}\right)=\vartheta(\rceil_{2}\right), \vartheta^{\prime}(\rceil_{1}\right)=\vartheta^{\prime}(\rceil_{2}\right)
$$

By using this equalities we find

$$
\left.\left.W(u, \vartheta ;\urcorner_{1}\right)-W(u, \vartheta ;\urcorner_{2}\right)=0
$$

Similarly by using the transmission conditions $(4)-(7)$ we obtain

$$
W\left(u, \vartheta ; c_{1}^{-}\right)-W\left(u, \vartheta ; c_{1}^{+}\right)=0
$$


and

$$
W\left(u, \vartheta ; c_{2}^{-}\right)-W\left(u, \vartheta ; c_{2}^{+}\right)=0
$$

Substituting the equalities (22), (23), (24) in (21) we get

$$
\int_{\tau_{1}}^{c_{1}^{-}}\left[\vartheta \Xi_{\lambda} u-u \Xi_{\lambda} \vartheta\right] d x+\int_{c_{1}^{+}}^{c_{2}^{-}}\left[\vartheta \Xi_{\lambda} u-u \Xi_{\lambda} \vartheta\right] d x+\int_{c_{2}^{+}}^{\urcorner_{2}}\left[\vartheta \Xi_{\lambda} u-u \Xi_{\lambda} \vartheta\right] d x=0
$$

which completes the proof.

\section{The Transmittal-Characteristic Function}

Consider the initial value problem

$$
\begin{gathered}
-y^{\prime \prime}+q(x) y=\lambda y, x \in\left(\alpha_{1}, \alpha_{2}\right) \\
y(\alpha+0)=r(\lambda), y^{\prime}(\alpha+0)=s(\lambda)
\end{gathered}
$$

where $r, s: \mathbb{C} \rightarrow \mathbb{C}$ are given complex functions. Using the method in [29], we can prove the following Lemma.

Lemma 3.1. Assume that the real valued function $q(x)$ is continuous on $\left(\alpha_{1}, \alpha_{2}\right)$ and the complex functions $r(\lambda), s(\lambda)$ are differentiable on whole complex plane $\mathbb{C}($ i.e. $r(\lambda)$ and $s(\lambda)$ are entire functions). Then, the initial value problem (25)-(26) has an unique solution $y=y(x, \lambda)$ which is an entire function of $\lambda$ for each fixed $x \in\left(\alpha_{1}, \alpha_{2}\right)$.

Let us construct two basic solutions

$$
\varphi(x, \lambda)=\left\{\begin{array}{ll}
\varphi_{1}(x, \lambda), & \left.x \in[\urcorner_{1}, c_{1}\right) \\
\varphi_{2}(x, \lambda), & x \in\left(c_{1}, c_{2}\right) \\
\varphi_{3}(x, \lambda), & \left.x \in\left(c_{2},\right\urcorner_{2}\right]
\end{array}, \chi(x, \lambda)= \begin{cases}\chi_{1}(x, \lambda), & \left.x \in[\urcorner_{1}, c_{1}\right) \\
\chi_{2}(x, \lambda), & x \in\left(c_{1}, c_{2}\right) \\
\chi_{3}(x, \lambda), & \left.x \in\left(c_{2},\right\urcorner_{2}\right]\end{cases}\right.
$$

according to the following iterative technique. First, we define the solution $\varphi_{1}(x, \lambda)$. Let $\varphi_{1}(x, \lambda)$ be the solution of the equation (1) on $\left.\Omega_{1}:=[\rceil_{1}, c_{1}\right)$ subject to the initial conditions

$$
\left.\left.y(\urcorner_{1}\right)=1, \quad y^{\prime}(\urcorner_{1}\right)=0
$$

Second, we shall define the solution $\varphi_{2}(x, \lambda)$ of Eq. (1) on $\Omega_{2}:=\left(c_{1}, c_{2}\right)$ by means of the solution $\varphi_{1}(x, \lambda)$ chosen so as to satisfy the initial conditions

$$
y\left(c_{1}^{+}\right)=\varphi_{1}\left(c_{1}^{-}, \lambda\right), \quad y^{\prime}\left(c_{1}^{+}\right)=\varphi_{1}^{\prime}\left(c_{1}^{-}, \lambda\right)
$$

Finally, we can define the solution $\varphi_{3}(x, \lambda)$ of Eq. (1) on $\left.\Omega_{3}:=\left(c_{2},\right\rceil_{2}\right]$ by means of the solution $\varphi_{2}(x, \lambda)$ satisfying the initial conditions

$$
y\left(c_{2}^{+}\right)=\frac{1}{\beta} \varphi_{2}\left(c_{2}^{-}, \lambda\right), \quad y^{\prime}\left(c_{2}^{+}\right)=\beta \varphi_{2}^{\prime}\left(c_{2}^{-}, \lambda\right) .
$$

Using the same iterative technique as in defining the solutions $\varphi_{1}(x, \lambda), \varphi_{2}(x, \lambda)$ and $\varphi_{3}(x, \lambda)$, we construct other solutions $\chi_{1}(x, \lambda), \chi_{2}(x, \lambda)$ and $\chi_{3}(x, \lambda)$ as a solution to the Eq.(1) chosen as to satisfy the initial conditions

$$
\begin{gathered}
\left.\left.y(\urcorner_{1}\right)=0, \quad y^{\prime}(\urcorner_{1}\right)=1 \\
y\left(c_{1}^{+}\right)=\chi_{1}\left(c_{1}^{-}, \lambda\right), \quad y^{\prime}\left(c_{1}^{+}\right)=\chi_{1}^{\prime}\left(c_{1}^{-}, \lambda\right) .
\end{gathered}
$$


and

$$
y\left(c_{2}^{+}\right)=\frac{1}{\beta} \chi_{2}\left(c_{2}^{-}, \lambda\right), \quad y^{\prime}\left(c_{2}^{+}\right)=\beta \chi_{2}^{\prime}\left(c_{2}^{-}, \lambda\right)
$$

respectively. By virtue of the Lemma 3.1, each of the solutions $\varphi_{i}(x, \lambda)$ and $\chi_{i}(x, \lambda)(i=1,2,3)$ exists, unique for any fixed $\lambda$ and is an entire function with respect to the complex variable $\lambda$ for any fixed $x$.

Theorem 3.2. Each of the pair $\varphi_{i}(x, \lambda), \chi_{i}(x, \lambda)$ is linearly independent solutions of Eq.(1) on the interval $\Omega_{i}$, where $\left.\left.\Omega_{1}=[\rceil_{1}, c_{1}\right), \Omega_{2}=\left(c_{1}, c_{2}\right), \Omega_{3}=\left(c_{2},\right\urcorner_{2}\right]$.

Proof. To prove it is sufficient to show that the Wronskians

$$
W_{\lambda}\left(\varphi_{i}, \chi_{i} ; x\right)=: \varphi_{i}(x, \lambda) \chi_{i}^{\prime}(x, \lambda)-\varphi_{i}^{\prime}(x, \lambda) \chi_{i}(x, \lambda)
$$

are not equal to zero on $\Omega_{i}$.

Since the Wronskians $W_{\lambda}\left(\varphi_{i}, \chi_{i} ; x\right)$ does not depend on variable $x$, Using the initial conditions (27 and (30) we have

$$
\left.W_{\lambda}\left(\varphi_{1}, \chi_{1} ; x\right)=W_{\lambda}\left(\varphi_{1}, \chi_{1} ;\right\urcorner_{1}\right)=1 \neq 0 .
$$

Using (28), (31) and (33)

$$
\begin{aligned}
W_{\lambda}\left(\varphi_{2}, \chi_{2} ; x\right) & =W_{\lambda}\left(\varphi_{2}, \chi_{2} ; c_{1}^{+}\right) \\
& =\varphi_{2}\left(c_{1}^{+}, \lambda\right) \chi_{2}^{\prime}\left(c_{1}^{+}, \lambda\right)-\varphi_{2}^{\prime}\left(c_{1}^{+}, \lambda\right) \chi_{2}\left(c_{1}^{+}, \lambda\right) \\
& =\varphi_{1}\left(c_{1}^{-}, \lambda\right) \chi_{1}^{\prime}\left(c_{1}^{-}, \lambda\right)-\varphi_{1}^{\prime}\left(c_{1}^{-}, \lambda\right) \chi_{1}\left(c_{1}^{-}, \lambda\right) \\
& \left.=W_{\lambda}\left(\varphi_{1}, \chi_{1} ; c_{1}^{-}\right)=W_{\lambda}\left(\varphi_{1}, \chi_{1} ;\right\urcorner_{1}\right)=1 \neq 0 .
\end{aligned}
$$

Similarly, using (29), (32) and 34) we get

$$
\begin{aligned}
W_{\lambda}\left(\varphi_{3}, \chi_{3} ; x\right) & =W_{\lambda}\left(\varphi_{3}, \chi_{3} ; c_{2}^{+}\right) \\
& =\varphi_{3}\left(c_{2}^{+}, \lambda\right) \chi_{3}^{\prime}\left(c_{2}^{+}, \lambda\right)-\varphi_{3}^{\prime}\left(c_{2}^{+}, \lambda\right) \chi_{3}\left(c_{2}^{+}, \lambda\right) \\
& =\left(\frac{1}{\beta} \varphi_{2}\left(c_{2}^{-}, \lambda\right)\right)\left(\beta \chi_{2}^{\prime}\left(c_{2}^{-}, \lambda\right)\right)-\left(\beta \varphi_{2}^{\prime}\left(c_{2}^{-}, \lambda\right)\right)\left(\frac{1}{\beta} \chi_{2}\left(c_{2}^{-}, \lambda\right)\right) \\
& =W_{\lambda}\left(\varphi_{2}, \chi_{2} ; c_{2}^{-}\right)=1 \neq 0 .
\end{aligned}
$$

The proof is complete.

Theorem 3.3. A complex number $\lambda$ is an eigenvalue of the transmittal-periodic problem (1)-(7) if and only if

$$
\left.\left.\left.\Delta(\lambda):=W_{\lambda}\left(\varphi_{2}, \chi_{2} ; c_{2}^{+}\right) W_{\lambda}\left(\varphi_{2}, \chi_{2} ; c_{2}^{-}\right)\left[W_{\lambda}\left(\varphi_{3}, \chi_{3} ;\right\urcorner_{1}\right)+1-\varphi_{3}(\urcorner_{2}\right)-\chi_{3}^{\prime}(\urcorner_{2}\right)\right]=0 .
$$

Proof. Let $y_{0}\left(x, \lambda_{0}\right)$ be any eigenfunction belonging to the eigenvalue $\lambda_{0}$. It follows from the Theorem 3.2 that the solutions $\varphi_{i}\left(x, \lambda_{0}\right)$ and $\chi_{i}\left(x, \lambda_{0}\right)$ are linearly independent solutions of $(1)$ on the $\Omega_{i} i=$ $1,2,3$. Therefore the eigenfunction $y_{0}\left(x, \lambda_{0}\right)$ may be represented as

$$
y_{0}\left(x, \lambda_{0}\right)=\left\{\begin{array}{l}
\delta_{1} \varphi_{1}\left(x, \lambda_{0}\right)+\gamma_{1} \chi_{1}\left(x, \lambda_{0}\right) \text { for } x \in \Omega_{1} \\
\delta_{2} \varphi_{2}\left(x, \lambda_{0}\right)+\gamma_{2} \chi_{2}\left(x, \lambda_{0}\right) \text { for } x \in \Omega_{2} \\
\delta_{3} \varphi_{2}\left(x, \lambda_{0}\right)+\gamma_{3} \chi_{2}\left(x, \lambda_{0}\right) \text { for } x \in \Omega_{3}
\end{array}\right.
$$

where at least one of the coefficients $\delta_{1}, \delta_{2}, \delta_{3}, \gamma_{1}, \gamma_{2}, \gamma_{3}$ is not zero. Now applying the boundary and transmission conditions (2)-(7) we obtain

$$
\ell_{i} y_{0}\left(x, \lambda_{0}\right)=0, i=1,2,3,4,5,6
$$


These equalities forms a homogeneous linear system of algebraic equations with respect to the variables $\delta_{1}, \delta_{2}, \delta_{3}, \gamma_{1}, \gamma_{2}, \gamma_{3}$ whose determinant has the form

$$
\Delta(\lambda):=\left|\begin{array}{cccccc}
1 & 0 & 0 & 0 & \left.-\varphi_{3}(\urcorner_{2}\right) & \left.-\chi_{3}(\urcorner_{2}\right) \\
0 & 1 & 0 & 0 & \left.-\varphi_{3}^{\prime}(\urcorner_{2}\right) & \left.-\chi_{3}^{\prime}(\urcorner_{2}\right) \\
\varphi_{2}\left(c_{1}^{+}\right) & \chi_{2}\left(c_{1}^{+}\right) & -\varphi_{2}\left(c_{1}^{+}\right) & -\chi_{2}\left(c_{1}^{+}\right) & 0 & 0 \\
\varphi_{2}^{\prime}\left(c_{1}^{+}\right) & \chi_{2}^{\prime}\left(c_{1}^{+}\right) & -\varphi_{2}^{\prime}\left(c_{1}^{+}\right) & -\chi_{2}^{\prime}\left(c_{1}^{+}\right) & 0 & 0 \\
0 & 0 & \varphi_{2}\left(c_{2}^{-}\right) & \chi_{2}\left(c_{2}^{-}\right) & -\varphi_{2}\left(c_{2}^{-}\right) & -\chi_{2}\left(c_{2}^{-}\right) \\
0 & 0 & \varphi_{2}^{\prime}\left(c_{2}^{-}\right) & \chi_{2}^{\prime}\left(c_{2}^{-}\right) & -\varphi_{2}^{\prime}\left(c_{2}^{-}\right) & -\chi_{2}^{\prime}\left(c_{2}^{-}\right)
\end{array}\right|
$$

It is easy to show that

$$
\left.\left.\left.\Delta(\lambda)=W_{\lambda}\left(\varphi_{2}, \chi_{2} ; c_{2}^{+}\right) W_{\lambda}\left(\varphi_{2}, \chi_{2} ; c_{2}^{-}\right)\left[W_{\lambda}\left(\varphi_{3}, \chi_{3} ;\right\urcorner_{2}\right)+1-\varphi_{3}(\urcorner_{2}\right)-\chi_{3}^{\prime}(\urcorner_{2}\right)\right]
$$

Since the system of algebraic linear equations (37) has nontrivial solution, we have $\Delta\left(\lambda_{0}\right)=0$. Now, show that any zero $\lambda=\lambda_{0}$ of the function $\Delta(\lambda)$ is an eigenvalue of the considered problem (1)-(7). Indeed, if $\Delta\left(\lambda_{0}\right)=0$, then the system (37) has a nontrivial solution $\left(\delta_{1}, \gamma_{1}, \delta_{2}, \gamma_{2}, \delta_{3}, \gamma_{3}\right)$. Therefore the nonrivial function $y_{0}\left(x, \lambda_{0}\right)$ defined by (36) satisfies the equation (1) and the boundary and transmission conditions (2)-(7). This means, that $\lambda_{0}$ is an eigenvalue.

Definition 3.4. The function $\Delta(\lambda)$ defined by

$$
\left.\left.\left.\Delta(\lambda)=W_{\lambda}\left(\varphi_{2}, \chi_{2} ; c_{2}^{+}\right) W_{\lambda}\left(\varphi_{2}, \chi_{2} ; c_{2}^{-}\right)\left[W_{\lambda}\left(\varphi_{3}, \chi_{3} ;\right\urcorner_{1}\right)+1-\varphi_{3}(\urcorner_{2}\right)-\chi_{3}^{\prime}(\urcorner_{2}\right)\right]
$$

will be called the transmittal-characteristic function for the boundary value problem (1)-(7).

Corollary 3.5. The transmittal-characteristic function $\Delta(\lambda)$ is an entire function.

\section{Conclusion}

This work is devoted to the spectral analysis of Sturm-Liouville problems of a new type. In fact, we studied three different Sturm-Liouville equations for three unknown solutions, which are defined on three disjoint subintervals, at the common ends of which four interaction conditions are imposed, the so-called transmission conditions. We first established that the eigenvalues are real and the corresponding eigenfunctions are orthogonal in the appropriate Hilbert space. It is also shown that the considered boundary value problem generates a self-adjoint linear differential operator. Second, using our own approach, we constructed special one-interval solutions, in terms of which a characteristic function of a new type is defined, the so called the transmittal-characteristic function. Finally, we proved that the eigenvalues coincide with the zeros of this characteristic function, which is an entire function. The results obtained are a generalization of the analogous classical results, since in the particular case $\beta=1$ our results are equivalent to the analogous results for the classical SturmLiouville problems.

\section{Author Contributions}

All the authors contributed equally to this work. They all read and approved the last version of the manuscript.

\section{Conflicts of Interest}

The authors declare no conflict of interest.

\section{Acknowledgement}

This work was supported by the Office of Scientific Research Projects Coordination at Amasya University, Grant number: FMB-BAP 20-0440. 


\section{References}

[1] İ. Çelik, G. Gökmen, Approximate Solution of Periodic Sturm-Liouville Problems with Chebyshev Collocation Method, Applied Mathematics and Computation 170(1) (2005) 285-295.

[2] X. Haoa, L. Liu, Y. Wu, Existence and Multiplicity Results for Nonlinear Periodic Boundary Value Problems, Nonlinear Analysis: Theory, Methods and Applications 72(9-10) (2010) 3635-3642.

[3] J. Li, J. J. Nieto, J. Shen, Impulsive Periodic Boundary Value Problems of First-Order Differential Equations, Journal of Mathematical Analysis and Applications 325(1) (2007) 226-236.

[4] J. Qiu, Positive Solutions for a Nonlinear Periodic Boundary-Value Problem with a Parameter, Electronic Journal of Differential Equations 2012(133) (2012) 1-10.

[5] G. V. Berghe, M. V. Daele, H. D. Meyer, A Modified Difference Scheme for Periodic and Semiperiodic Sturm-Liouville Problems, Applied Numerical Mathematics 18(1-3) (1995) 69-78.

[6] P. A. Binding, P. B. Rynne, Half-Eigenvalues of Periodic Sturm-Liouville Problems, Journal of Differential Equations 206(2) (2004) 280-305.

[7] P. Binding, H. Volkmer, A rüfer Angle Approach to the Periodic Sturm-Liouville Problem, The American Mathematical Monthly 119(6) (2012) 477-484.

[8] A. Boumenir, Eigenvalues of Periodic Sturm-Liouville Problems by the Shannon-Whittaker Sampling Theorem, Mathematics of Computation of the American Mathematical Society 68(227) (1999) 1057-1066.

[9] F. Geng, Y. Xu, D. Zhu, Periodic Boundary Value Problems for First-Order Impulsive Dynamic Equations on Time Scales, Nonlinear Analysis: Theory, Methods and Applications 69(11) (2008) 4074-4087.

[10] K. V. Khmelnytskaya, H. Rosu, C. A. González, Periodic Sturm-Liouville Problems Related to Two Riccati Equations of Constant Coefficients, Annals of Physics 325(3) (2010) 596-606.

[11] V. A. Sadovnichii, Y. T. Sultanaev, A. M. Akhtyamov, Inverse Sturm-Liouville Problem with Generalized Periodic Boundary Conditions, Differential Equations 45(4) (2009) 526-538.

[12] A. A. Shkalikov, O. A. Veliev, On the Riesz Basis Property of the Eigen-and Associated Functions of Periodic and Antiperiodic Sturm-Liouville Problems, Mathematical Notes 85(5-6) (2009) 647660.

[13] S. Somali, V. Oger, Improvement of Eigenvalues of Sturm-Liouville Problem with t-Periodic Boundary Conditions, Journal of Computational and Applied Mathematics 180(2) (2005) 433441.

[14] Y. Yuan, J. Sun, A. Zettl, Eigenvalues of Periodic Sturm Liouville Problems, Linear Algebra and Its Applications 517 (2017) 148-166.

[15] Y. Wang, J. Li, Z. Cai. Positive Solutions of Periodic Boundary Value Problems for the SecondOrder Differential Equation with a Parameter, Boundary Value Problems Article Number 49 2017(1) (2017) 1-11.

[16] Y. Zhao, H. Chen, B. Qin, Periodic Boundary Value Problems for Second-Order Functional Differential Equations with Impulse, Advances in Difference Equations Article Number 134 2014(1) (2014) 1-12.

[17] I. Akbarfam, A. Jodayree, Resolvent Operator and Self-Adjointness of Sturm-Liouville Operators with a Finite Number of Transmission Conditions, Mediterranean Journal Of Mathematics 11(2) (2014) 447-462. 
[18] B. P. Allahverdiev, E. Bairamov, E. Ugurlu, Eigenparameter Dependent Sturm-Liouville Problems in Boundary Conditions with Transmission Conditions, Journal of Mathematical Analysis and Applications 401(1) (2013) 388-396.

[19] B. P. Allahverdiev, H. Tuna, On the Resolvent of Singular Sturm-Liouville Operators with Transmission Conditions, Mathematical Methods in The Applied Sciences 43(7) (2020) 388-396.

[20] J. Ao, J. Sun, Eigenvalues of a Class of Fourth-Order Boundary Value Problems with Transmission Conditions Using Matrix Theory, Linear and Multilinear Algebra 69(9) (2021) 1610-1624.

[21] K. Aydemir, H. Olğar, O. Sh. Mukhtarov, Differential Operator Equations with Interface Conditions in Modified Direct Sum Spaces, Filomat 32(3) (2018) 921-931.

[22] E. Bairamov, E. Uğurlu, On the Characteristic Values of the Real Component of a Dissipative Boundary Value Transmission Problem, Applied Mathematics and Computation 218(19) (2012) 9657-9663.

[23] H. Olğar, O. Sh. Mukhtarov, Weak Eigenfunctions Of Two-Interval Sturm-Liouville Problems Together With Interaction Conditions, Journal of Mathematical Physics 58(042201) (2017) DOI: 10.1063/1.4979615.

[24] H. Olğar, F. Muhtarov, The Basis Property of the System of Weak Eigenfunctions of a Discontinuous Sturm-Liouville Problem, Mediterranean Journal of Mathematics Article Number 114 14(3) (2017) 1-13.

[25] O. Sh. Mukhtarov, K. Aydemir, Discontinuous Sturm-Liouville Problems Involving an Abstract Linear Operator, Journal of Applied Analysis and Computation 10(4) (2020) 1545-1560.

[26] O. Sh. Mukhtarov, H. Olğar, K. Aydemir, I. Sh. Jabbarov, Operator-Pencil Realization of One Sturm-Liouville Problem with Transmission Conditions, Applied and Computational Mathematics 17(2) (2018) 284-294.

[27] O. Sh. Mukhtarov, M. Yücel, K. Aydemir, A New Generalization of the Differential Transform Method for Solving Boundary Value Problems, Journal of New Results in Science 10(2) (2021) $49-58$.

[28] E. Şen, Sturm-Liouville Problems With Retarded Argument and A Finite Number of Transmission Conditions, Electronic Journal Of Differential Equations 2017(310) (2017) 1-8.

[29] E. C. Titchmarsh, Eigenfunctions Expansion Associated with Second Order Differential Equations I, Second edn. Oxford University Press, London, 1962. 